\title{
Frequency Tuning of a Disk Resonator Gyro via Mass Matrix Perturbation
}

\author{
David Schwartz, Dong Joon Kim, Robert T. M'Closkey, Member IEEE
}

\begin{abstract}
Electrostatic tuning of the resonant modes in vibratory gyroscopes is often suggested as a means for compensating manufacturing aberrations that produce detuned resonances. In high performance sensors, however, this approach places very stringent requirements on the stability of the bias voltages used for tuning. Furthermore, the bias voltage stability must be maintained over the operating environment, especially with regard to temperature variations. This paper presents two methods for tuning the resonant modes in MEM vibratory gyroscopes using mass perturbation of the sensor's resonant structure. The approach ameliorates the stringent bias voltage stability requirements and can be applied to any vibratory gyroscope that relies on modal frequency matching for optimum performance.
\end{abstract}

\section{INTRODUCTION}

Most high-performance vibratory angular rate sensors rely on the matching of the frequencies of two modes that are highly coupled by a Coriolis acceleration term when the equations of motion are written in a case-fixed coordinate system. Frequency matching exploits the mechanical gain afforded by the sensor dynamics and leads to the best attainable signal-to-electronic noise ratio. The degenerate dynamics can be attained by designing structures with a high degree of symmetry and in the case of the Boeing Silicon Disk Resonator Gyro (SiDRG) this symmetric design also provides a high degree of isolation of the Coriolis modes from linear acceleration of the sensor case. These ideas have also been exploited in Litton's Hemispherical Resonator Gyro (HRG), the BAE nickel ring gyro, and the BEI quartz tuning fork gyro. In all of these sensors, the anchor attaching the resonant structure to the sensor case is, ideally, a nodal point for the Coriolis coupled modes and the symmetric design, on its face, guarantees degenerate modal frequencies. Furthermore, the high degree of isolation reduces energy dissipation in the modes, which is a source of angular rate bias and drift. The HRG is an extreme example of the degree of isolation that can be achieved- quality factors exceeding $6 \times 10^{6}$ have been reported when the resonators are fabricated from fused quartz [6], [7].

For those sensors lending themselves to MEM fabrication, such as the SiDRG, local variations in etch rate produce very small, but unpredictable, asymmetries that manifest

Manuscript submitted on September 21, 2007. This work was supported by Boeing and NSF grant \#0601622.

The authors are with the Mechanical and Aerospace Engineering Department, University of California, Los Angeles, CA 90095 USA.

David Schwartz is a Graduate Student Researcher.

Dong Joon Kim is a Postdoctoral Researcher

Robert T. M'Closkey is Professor and corresponding author (310 8252909, rtm@obsidian. seas.ucla.edu)

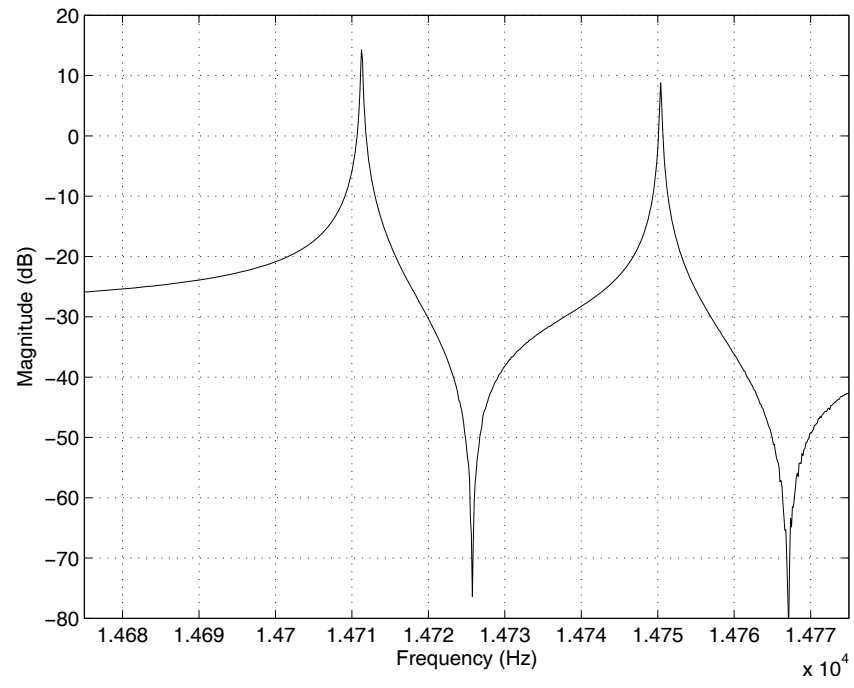

Fig. 1. SiDRG frequency response within a narrow, $100 \mathrm{~Hz}$ band encompassing the "fundamental" Coriolis modes. Though the frequency split is small in a relative sense -less than $0.3 \%$-the sensor effectively has no mechanical gain in this state. The present work proposes a systematic method for tuning these modal frequencies to degeneracy by perturbing the resonator mass matrix.

themselves as a splitting of the modal frequencies (Fig. 1). Although the frequency splits are small, on the order of $0.3 \%$ or less, the absolute separation between the modal frequencies coupled with their relatively high $\mathrm{Q}$ conspire to eliminate the mechanical gain advantage that was a primary objective of sensor's design in the first place.

In past work, the resonant frequencies of the SiDRG were tuned by locally altering the resonator stiffness by applying electrostatic forces with dedicated electrodes [2], [3], [5]. Unfortunately the electrodes are required to hold a very stable voltage over the operating environment, which is difficult to do with compact, low-cost electronics. The possibility of tuning the modes by permanently altering the mass distribution of the sensor is attractive because it eliminates the need for tuning bias voltages [1]. On the SiDRG this can be accomplished by using a laser to strategically trim the resonator. In order to develop the mass perturbation algorithms a scale model of the SiDRG, called the Macro DRG, was fabricated. Using a novel model identification method, the effects of mass matrix perturbation on the Macro DRG are studied and several frequency tuning approaches are demonstrated. 


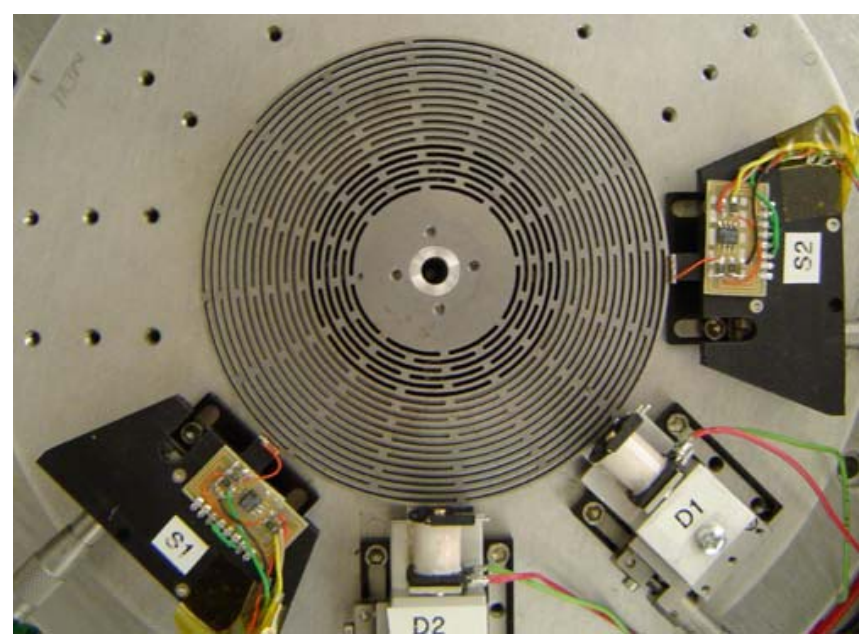

Fig. 2. Photograph of the Macro DRG. The two electromagnetic actuators are labeled $D_{1}$ and $D_{2}$, and the two capacitive pick-offs that detect planar deflection of the resonator are located at $S_{1}$ and $S_{2}$.

\section{MACRo DRG ExPerimental Setup}

The Boeing SiDRG was developed to exhibit the advantages of the HRG at a much lower cost. The end result is a thin concentric ring structure with an $8 \mathrm{~mm}$ diameter. Sensing, driving and trimming electrodes are embedded inside the gaps between the rings.

The Macro DRG, on the other hand, is a steel simulacrum of the SiDRG with an outer diameter of $12 \mathrm{~cm}$ (Fig. 2). The Macro DRG is instrumented with two electromagnetic actuators and two capacitive sensing pick-offs that are mounted on linear translation stages for a precise gap control. Fig. 3 shows the block diagram of a testing setup for an open loop system identification. A DSP generates signals that drive an op-amp whose ouput energizes the electromagnetic actuators thereby applying radial forces to the resonator. The structural response creates a varying capacitance that produces a current which is then converted into buffered voltage via a transimpedance amplifier. The sense signals are further amplified, scaled and filtered prior to sampling. Small NdFeB magnets (disc shape, $1.5 \mathrm{~mm}$ diameter, $.75 \mathrm{~mm}$ thickness) are placed on the top surface of the resonator to create (reversible) mass perturbations.

A single channel of Macro DRG frequency response data is shown in Fig. 4. The fundamental Coriolis modes are near $1.6 \mathrm{kHz}$ and the zoomed frequency response shows the individual Coriolis-coupled modes exhibiting a $2 \mathrm{~Hz}(0.12 \%)$ frequency split.

\section{SENSOR MOdeL}

The system identification method used to guide the mass perturbation process is based on the one developed by the authors for electrostatic tuning of the gyro dynamics [5]. The electrostatic tuning case will be briefly reviewed in order to highlight the differences with the sensor model used in the mass perturabation study. For electrostatic tuning, the linear mechanics of nearly degenerate vibratory gyros in a

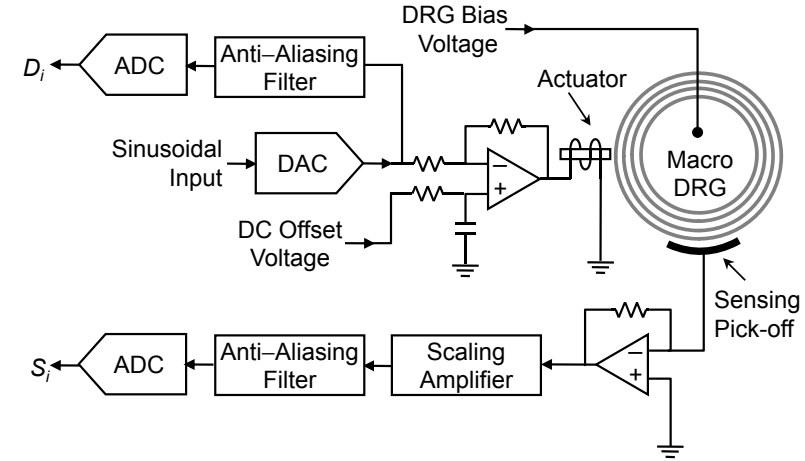

Fig. 3. Block diagram of test setup. Filtered drive and sense signals are denoted $D_{i}$ and $S_{i}, i=1,2$. Frequency response data is used to construct a two-input/two-output model of the sensor.

neighborhood of the Coriolis-coupled modes can be modeled as

$$
H_{\text {out }}(s) R Z_{\text {act }}^{-1}(s),
$$

where $s$ is the Laplace transform variable and where

$$
Z_{a c t}(s):=M s^{2}+C s+K_{0}+\sum_{p=1}^{n_{e}} K_{p} v_{p}^{2}
$$

In this model $M, C$, and $K_{0}$ are real $2 \times 2$ positive definite mass, damping and stiffness matrices, respectively. There are $n_{e}$ electrodes on the sensor that are dedicated for electrostatically tuning the modes and the "stiffness matrix sensitivity" associated with these electrodes are given by the real symmetric matrices $K_{p}, p=1, \ldots, n_{e}$, which are multiplied by the square of the voltage potentials existing between the electrodes and resonator (denoted $v_{p}, p=1, \ldots, n_{e}$ ). The angular rotation rate of the sensor is assumed to be zero in this model. The subscript on $Z_{a c t}$ denotes that the system matrices are written in the generalized coordinates specified by the actuator (forcer) frame. The transfer function $H_{\text {out }}$ represents any dynamics associated with the signal conditioning electronics and $R \in \mathbf{R}^{2 \times 2}$ captures the effects of noncolocated pick-offs and forcers.

The model parameters $\left\{M, C, K_{0}, K_{1}, \ldots, K_{n_{e}}\right\}$ are estimated by using frequency response data from $n_{\exp }$ experiments conducted at different tuning electrode voltage potentials. In other words, the $k$ th experiment with tuning potentials defined by $\left\{v_{k, 1}, v_{k, 2}, \ldots, v_{k, n_{e}}\right\}, k=1, \ldots, n_{\text {exp }}$, yields two-input/two-output complex-valued frequency response data $\left\{\Psi_{k, 1}, \Psi_{k, 2}, \ldots, \Psi_{k, m_{k}}\right\}$ corresponding to the $m_{k}$ frequencies $\left\{\omega_{k, 1}, \omega_{k, 2}, \ldots, \omega_{k, m_{k}}\right\}$.

The minimax optimization problem for estimating the sensor parameters is

$$
\min _{\substack{M>I, C>0 \\ K_{p} \leq 0, p=1, \ldots, n_{e} \\ K_{0}+\sum K_{p} v_{p}^{2}>0 \\ R_{l} \in \mathbf{C}^{2 \times 2}, l=0,1, \ldots, n_{R}}} \max _{\substack{k=1, \ldots, n_{e x p} \\ q=1, \ldots, m_{k}}} \bar{\sigma}\left(\tilde{R}_{k, q}-\psi_{k, q} Z_{a c t}\left(j \omega_{k, q}\right)\right),
$$

where

$$
\tilde{R}_{k, q}:=\sum_{l=0}^{n_{R}} R_{l} \omega_{k, q}^{l}
$$



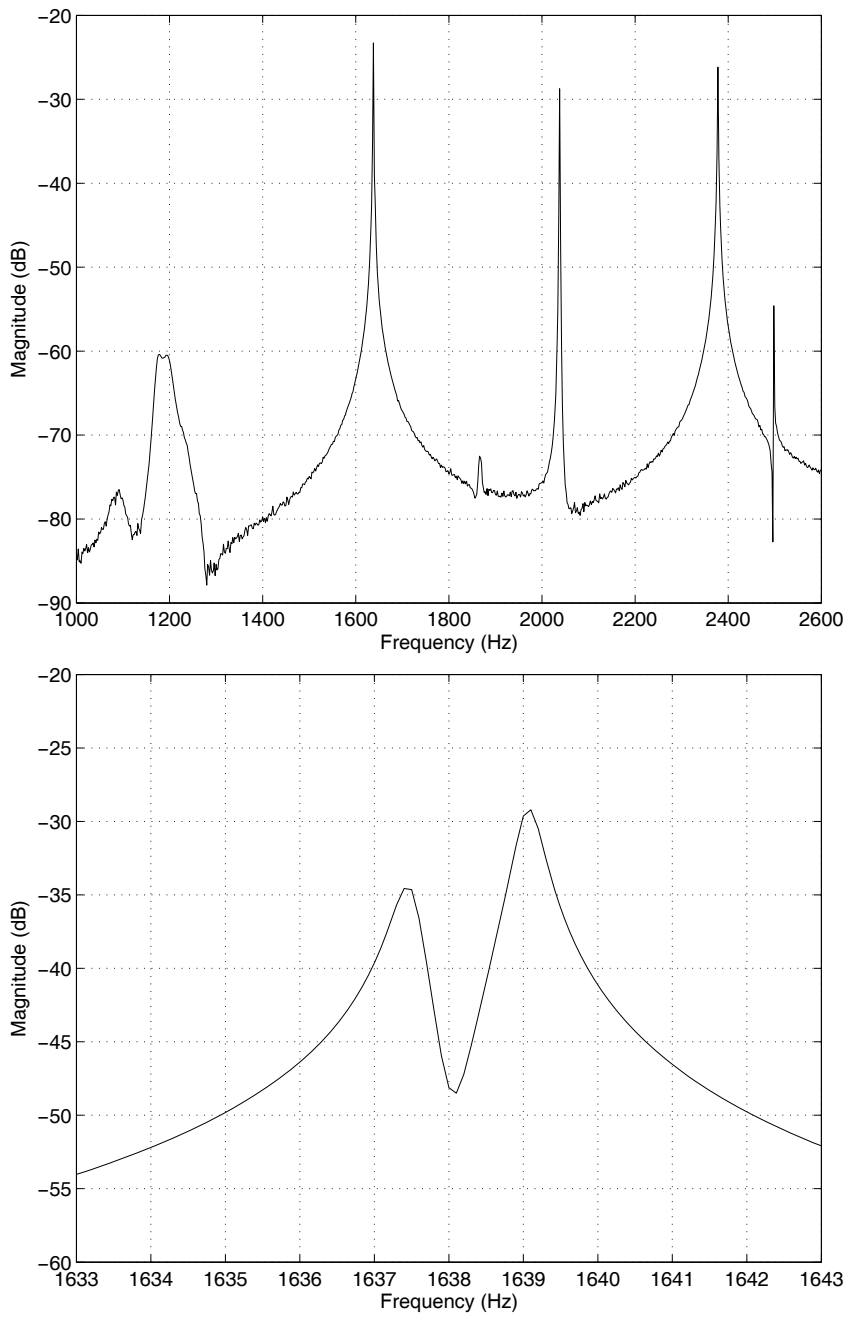

Fig. 4. The $S_{1} / D_{1}$ channel empirical frequency response of the Macro DRG showing wideband (top) and narrowband dynamics in a neighborhood of the Coriolis-coupled modes (bottom). Just as in the SiDRG response, the Coriolis-coupled modes of the Macro DRG have a small but detrimental frequency split despite the fact that the steel resonator visually exhibits symmetry. Small magnets are attached to the rings of the resonator to achieve mass loading, thereby altering the mass matrix governing the modal coupling.

and where evaluating $Z_{a c t}$ at the $q$ th frequency point associated with the $k$ th experiment yields

$$
Z_{a c t}\left(j \omega_{k, q}\right):=-M \omega_{k, q}^{2}+K_{0}+\sum_{p=1}^{n_{e}} K_{p} v_{k, p}^{2}+j C \omega_{k, q} .
$$

The $M>I$ constraint in (3) is imposed rather than the typical $M>0$ because in the latter case all of the free parameters may scaled by a nonzero constant so as to make the cost arbitrarily small without actually changing the model frequency response. Also note that $H_{\text {out }} R$ has been replaced by $\tilde{R}$. This recognizes the fact that any additional dynamics due to, for example, signal conditioning preamplifiers, should not exhibit significant magnitude and phase changes in a neighborhood of the resonant modes. If these dynamics can be reflected to the sensor output then they can be combined with $R$ into a low order polynomial function of frequency with coefficients in $\mathbf{C}^{2 \times 2}$, i.e. $\tilde{R}$ is degree $n_{R}$. In fact, $\tilde{R}$ can be viewed as combining the first few terms of the Taylor series expansion of the frequency response function of $H_{\text {out }}$ with sensor-actuator combined with non-collocation effects. Once the model parameters are identified it is possible to compute the bias potentials necessary to tune the modes to degeneracy, i.e. the potentials are selected so that the generalized eigenvalues of the pair $\left(M, K_{0}+\sum_{p=1}^{n_{e}} K_{p} v_{p}^{2}\right)$ are equal.

The present study requires that we modify $Z_{a c t}$. Assume that $n_{\text {exp }}$ experiments are conducted in which mass is added to, or, removed from, the resonator. The $k$ th experiment generates frequency response data $\left\{\Psi_{k, 1}, \Psi_{k, 2}, \ldots, \Psi_{k, m_{k}}\right\}$ at the following grid of frequencies $\left\{\omega_{k, 1}, \omega_{k, 2}, \ldots, \omega_{k, m_{k}}\right\}$. The index $k=0$ is reserved for the unperturbed case. In this scenario, $Z_{a c t}$ in (3) is replaced by

$$
Z_{a c t}\left(j \omega_{k, q}\right):=-M_{k} \omega_{k, q}^{2}+K+j C \omega_{k, q}, \quad k=0,1, \ldots, n_{\text {exp }},
$$

where $\omega_{k, q}$ is the $q$ th frequency point affiliated with the $k$ th experiment. As mentioned above, $M_{0}$ represents the mass matrix of the unperturbed sensor, and $M_{1}, M_{2}$, etc., represent the mass matrices corresponding to perturbed sensor states. No distinction need be made between mass removal or mass addition. As in the electrostatic tuning case, a minimax problem can be specified to compute the model parameters in this mass perturbation scenario,

$$
\min _{\substack{K>0, C>0 \\ M_{k}>I, k=0,1, \ldots, n_{\text {exp }} \\ R_{l} \in \mathbf{C}^{2 \times 2}, l=0,1, \ldots, n_{R}}} \max _{q=1, \ldots, m_{k}} \bar{\sigma}\left(\tilde{R}_{k, q}-\psi_{k, q} Z_{a c t}\left(j \omega_{k, q}\right)\right),
$$

where $\tilde{R}_{k, q}$ is given by (4) and $\bar{\sigma}$ is the maximum singular value. The approach forces the differences between the frequency response data among the experiments to be captured by the changes in the sensor mass matrix. Note that (7) can be formulated as a convex optimization.

\section{EXPERIMENTAL RESULTS}

Experimental data is generated by driving each actuator with a narrow band chirp sequence that encompasses the Coriolis modes of the Macro DRG. The input-output sequences are processed to yield $2 \times 2$ empirical frequency response data on a grid of frequencies with $0.1 \mathrm{~Hz}$ resolution. The model (6) is now applied to two mass perturbation cases, in addition to the nominal case when no mass perturbation is present. The first mass perturbation case places four magnets on outer ring of the Macro DRG as shown in the left-hand picture of Fig. 5. The orientation of this picture coincides with the photograph in Fig. 2. The second mass perturbation case places four magnets on the outer ring of the DRG as shown in the right-hand picture of Fig. 5. The empirical frequency responses for these perturbed cases along with the unperturbed case (no magnets added) are shown as the top grouping of plots in Fig. 6. Since the sensor is a twoinput/two-output plant, four frequency response magnitude plots are shown in this figure (the individual channels are denoted $S_{1} / D_{1}, S_{2} / D_{1}$, etc.). It is clear that mass perturbations 

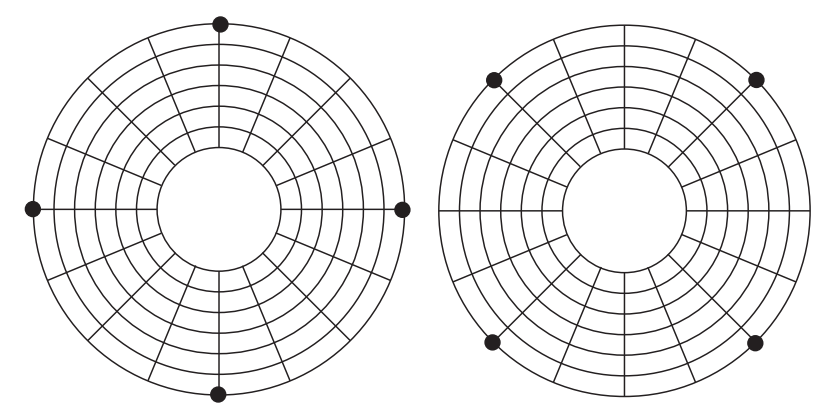

Fig. 5. Left. Location of $\Delta_{1}$ perturbation. Right. Location of $\Delta_{2}$ perturbation. Masses are added at four points in each $\Delta$ to achieve more even mass loading.

shift both modal frequencies, change the frequency split, and also modify the coupling between the channels. The modal parameter set $\left\{M_{0}, M_{1}, M_{2}, C, K\right\}$ is determined using (7) and the three sets of frequency response data corresponding the unperturbed sensor and the two perturbed cases are generated for comparison with the data. The model frequency response using mass matrix $M_{k}, k=0,1,2$, is given by $\left(\sum_{l=0}^{n_{R}} R_{l} \omega^{l}\right)\left(-M_{k} \omega^{2}+K+j C \omega\right)^{-1}$. Instead of plotting the model frequency response against the empirical frequency response, we plot the absolute error between the data and model,

$$
\bar{\sigma}\left(\Psi_{k, q}-\tilde{R}_{k, q} Z_{\text {act }}^{-1}\left(j \omega_{k, q}\right)\right), \quad k=0,1,2,
$$

These plots give direct assessment of the modeling error as function of frequency and are shown in the bottom plot in Fig. 6. The absolute error is approximately $25 \mathrm{~dB}$ smaller than the frequency response magnitude across the band of interest thereby demonstrating a good model fit. It is convenient to define the mass matrix perturbation as $\Delta_{k}=M_{k}-M_{0}, k=$ $1, \ldots, n_{\exp }$, so we will adopt this notation forthwith.

Since we are interested in using this model to guide the mass addition/removal process with the objective of driving the two modal frequencies together, the predictive power of the model is tested on two new data sets. The first test adds two magnets at each of the four points on the resonator corresponding to the positions shown the left-hand picture in Fig. 5 (total of eight magnets). This perturbation doubles the magnitude of the mass perturbation corresponding to $\Delta_{1}$ so we compare the empirical data with the frequency response of the model

$$
\left(\sum_{l=0}^{n_{R}} R_{l} \omega^{l}\right)\left(-\left(M_{0}+2 \Delta_{1}\right) \omega^{2}+K+j \omega C\right)^{-1} .
$$

The comparison is made in the top set of plots in Fig. 7 ("Case 1"). The model predicts the actual frequency response extremely well especially since the positioning of the magnets is done by hand.

The second test case involves placing a single magnet at each of the eight points on the resonator corresponding to the locations shown in both pictures in Fig. 5. This perturbation should correspond to modifying the nominal mass matrix
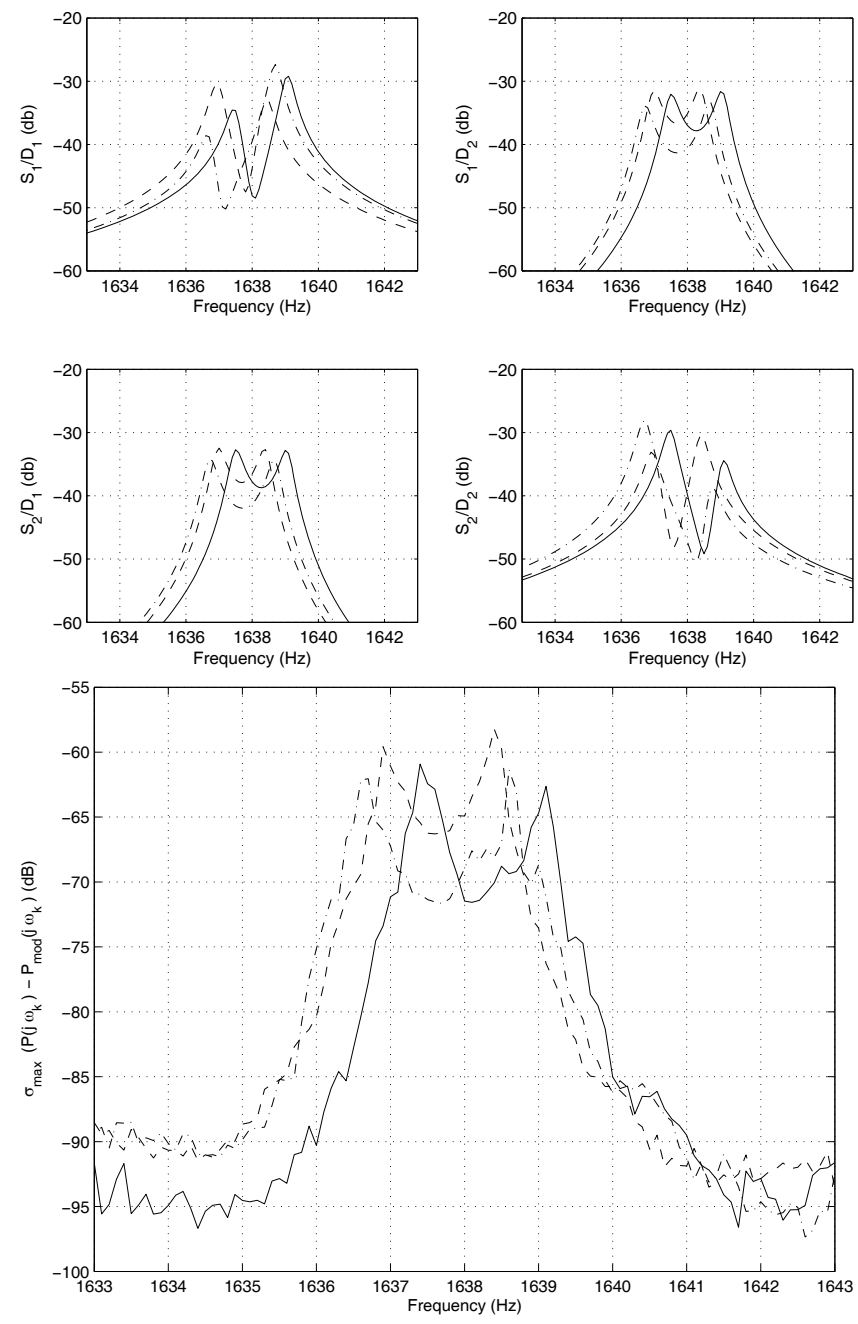

Fig. 6. (Top group) Empirical frequency response of Macro DRG in a neighborhood of the fundamental Coriolis coupled modes. The solid trace represents the native resonator dynamics without any mass perturbation. The other two dashed traces represent the resonator dynamics when mass perturbations are created by attaching small magnets to the resonator. The perturbation locations is shown in Fig. 5. (Bottom plot) Absolute error between the empirical frequency response data and the identified model.

by the sum of $\Delta_{1}$ and $\Delta_{2}$, thus we compare the frequency response data against

$$
\left(\sum_{l=0}^{n_{R}} R_{l} \omega^{l}\right)\left(-\left(M_{0}+\Delta_{1}+\Delta_{2}\right) \omega^{2}+K+j \omega C\right)^{-1} .
$$

Again, the bottom set of plots in Fig. 7 ("Case 2") shows a very good agreement between the model prediction and the empirical frequency response data.

\section{Tuning the Modal Frequencies}

The challenge with tuning the modal frequencies using the mass perturbation approach that is not present in the electrostatic tuning approach is the fact that the mass perturbation approach does not possess a simple representation of the mass perturbation matrix as a function of perturbation location on the resonator (recall that the tuning voltages 

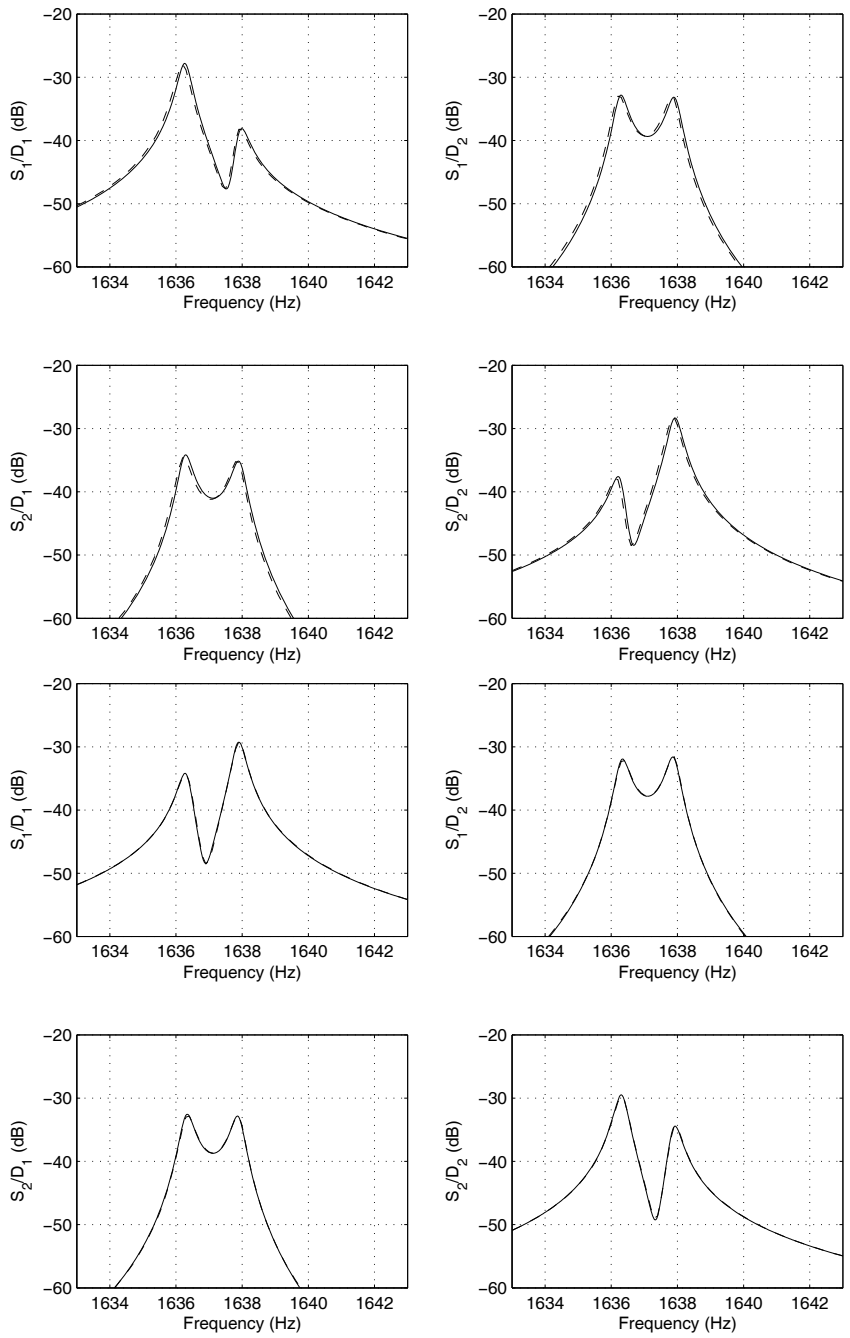

Fig. 7. Top: Empirical frequency response of macro DRG with double the mass perturbation at the $\Delta_{1}$ perturbation locations (dashed trace) compared to the frequency response predicted by the model $\tilde{R}(j \omega)\left(-\left(M_{0}+2 \Delta_{1}\right) \omega^{2}+\right.$ $K+j \omega C)^{-1}$ (thin solid trace). Bottom: Empirical frequency response of macro DRG with the mass perturbations in both the $\Delta_{1}$ and $\Delta_{2}$ perturbation locations (dashed trace) compared to the frequency response predicted by the model $\tilde{R}(j \omega)\left(-\left(M_{0}+\Delta_{1}+\Delta_{2}\right) \omega^{2}+K+j \omega C\right)^{-1}$ (thin solid trace).

appeared directly in the sensor model for the electrostatic case -the functional form of the stiffness matrix dependence on the tuning voltages is easily motivated by considering the energy stored in the capacitor formed by the resonator and tuning electrodes). It is possible to analytically derive a relationship between the perturbation location and the resulting mass matrix perturbation for a much simpler structure such as a single, thin ring. Unfortunately the complex topology of the DRG with its multiple, coupled, concentric rings precludes such an approach. The mass perturbation matrix can, however, be experimentally identified using the sensor model (6). Thus, we proceed to systematically observe the change in $M$ in one ninety degree arc at regular intervals (it can be shown that adding the same mass at 90 degree, or 180 degree, angles relative to the current position produces the same mass matrix perturbation for the modes of

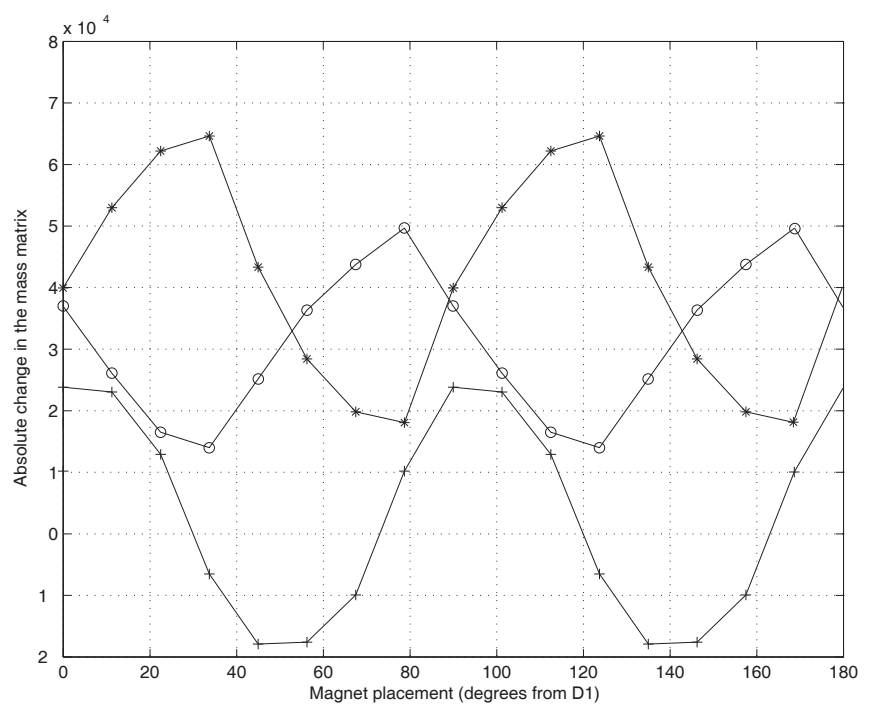

Fig. 8. Mass matrix perturbation, $\Delta$, as a function of the counter-clockwise angle from the $D_{1}$ axis, $\theta$, found by fitting the model to ten empirical frequency response data sets. The upper diagonal term is denoted by '*', the lower diagonal by 'o', and the off diagonal term by ' + '.

interest). Nine separate perturbations where conducted with magnets spaced in 11.25 degree steps (a total of ten MIMO frequency response data sets) and then the modal parameter set $\left\{M_{0}, M_{1}, \ldots, M_{9}, C, K\right\}$ was identified. A plot of the three components of each $\Delta_{k}, k=0,1, \ldots, 9$, is shown in Fig. 8 . The three traces represent the upper diagonal, lower diagonal and off diagonal terms of the positive definite $\Delta_{k}$ matrices.

We proceeded to use the identified model parameters to test different possible perturbation scenarios. For this study, linear interpolation is used to specify the perturbation matrix coefficients at angular values not represented in the experiments. This is clearly an approximation, but the guidance it provides in ultimately tuning the modes justifies its use. Thus, for our initial analysis we use the perturbed mass matrix

$$
M=M_{0}+\alpha \Delta(\theta)
$$

where $\Delta(\theta)$ represents the mass matrix perturbation as a function of angular perturbation location $\theta$ (in other words, the coefficients represented graphically in Fig. 8), and $\alpha$ represents the scalar perturbation magnitude (in our case with the magnets, the perturbation magnitude takes on discrete values since we can add only a whole number of magnets; on the other hand, there are other mass removal/deposition approaches that would provide much finer control over $\alpha$ ).

The objective is to find a perturbation magnitude and location that creates degenerate generalized eigenvalues of $M$ and $K$. In addition to changes in the generalized eigenvalues of $M$ and $K$ as a function of $\alpha$ and $\theta$, one can also observe changes in the generalized eigenvectors, which indicate the angular position of the anti-nodes of the modes. By analyzing the generalized eigenvalues we conclude that in the case of a single perturbation location (replicated with the 4-fold symmetry in the other quadrants vis-à-vis Fig. 5) there is 


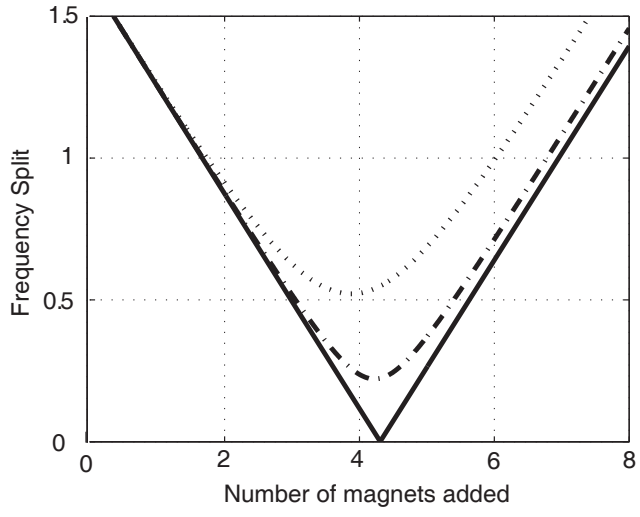

Fig. 9. Plots of the frequency split (in $\mathrm{Hz}$ ) predicted by the identified model as mass is added at seventy-five degrees (dotted line), eighty-two degrees (dash dotted line) and eighty degrees (solid line) measured counterclockwise from the $D_{1}$ actuator location. The frequency split is initially reduced in all three cases as mass is added, however, only when mass is added to a single ideal location can the resonant frequencies be completely tuned. This figure shows that the magnitude of the perturbation must also be carefully controlled.

a unique perturbation location and magnitude that tunes the modes. If the exact location is not correctly identified then it is not possible to tune the modes to degeneracy. Fig. 9 shows how the frequency split changes as function of perturbation location in a neighborhood of the location required for eigenvalue degeneracy. Thus, attempting to attain degeneracy by adding mass to only one angular location can be very difficult. It is possible, however, to attain convergence by adding mass to two different locations in a quadrant. By adding mass to either side of the anti-node of the higher frequency mode, the anti-nodal orientation is, in a sense, trapped. The anti-node is determined by the generalized eigenvector associated with the higher frequency mode. Since we are searching for a way to tune a MEM DRG, it will be easiest to add mass at the "spokes" of the resonator (visible in Fig. 2 as the structures that join adjacent rings and form a radial pattern) that are closest to the anti-node. In this scenario the perturbed mass matrix is given by $M=M_{0}+\alpha_{1} \Delta_{1}+\alpha_{2} \Delta_{2}$, where $\Delta_{1}$ and $\Delta_{2}$ correspond to the mass matrix perturbations associated with the desired spoke locations.

Testing these two scenarios on the Macro DRG was successful. Fig. 10 shows the empirical frequency responses of the tuned sensor using the mass matrix perturbations as function of $\alpha$ and $\theta$ as a guide. In one trace the DRG is tuned using only one angular location, and in the other trace the DRG is tuned using the two spoke approach. As expected, the off-diagonal channels exhibit reduced coupling and the diagonal frequency responses resemble a single degree of freedom resonator. Also, using the spokes for modal frequency tuning inevitably produces a smaller tuned frequency because more total mass is added to the resonator.
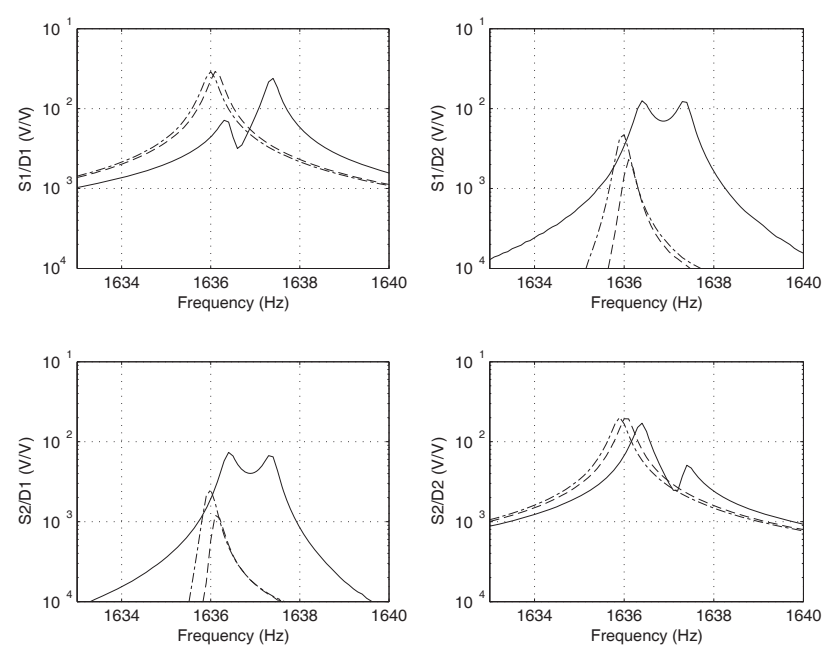

Fig. 10. Empirical frequency response of the Macro DRG tuned by single mass placement (dashed line) and the two spoke tuning approach (dash dotted line). The unperturbed frequency response is shown as the solid trace. Both approaches effectively tune the sensor modal frequencies to a frequency split of less than $100 \mathrm{mHz}$.

\section{Vi. Conclusions}

Two methods using a mass matrix perturbation approach for tuning the two Coriolis-coupled modes in a vibratory gyroscope have been experimentally demonstrated on a scale model of the Boeing Silicon Disk Resonator Gyro. Although mass was added to the resonator for this study, the methods are readily adapted to the situation in which mass is removed. Future work will address mass matrix perturbations that can reduce the coupling of linear acceleration to the Corioliscoupled modes. The latter objective is important for isolating the modes used for angular rate sensing from linear accleration of the sensor case.

\section{REFERENCES}

[1] Abdelmoneum, M.A, Demirci, M.M., Lin, Y-W., and Nguyen, C.T., "Location-Dependent Frequency Tuning of Vibrating Micromechanical Resonators Via Laser Trimming," 2004 IEEE Int. Ultrasonics, Ferroelectrics, and Frequency Control Symp., pp. 272-279, 2004.

[2] Adams, S.G., Bertsch, F.M., Shaw, K.A., and MacDonald, N.C., "Independent Tuning of Linear and Nonlinear Stiffness Coefficients," IEEE J. Microelectromechanical Systems, Vol. 7, No. 2, pp. 172-180, 1998.

[3] Ayazi, F., and Najafi, K., "A HARPS Polysilicon Vibrating Ring Gyroscope," IEEE J. Microelectromechanical Systems, Vol. 10, No. 2, pp. 169-179, 2001.

[4] Enderling, S., Hedley, J., Jiang, L., Cheung, R., Zorman, C., Mehregany, M., and Walton, A., "Characterization of frequency using focused ion beam platinum deposition," J. Micromech. Microeng., Vol. 7, pp. 213-219, 2007.

[5] Kim, D-J., and M'Closkey, R.T., "A systematic method for tuning the dynamics of electrostatically actuated vibratory gyros," IEEE Trans. Control System Technology, Vol. 14, No. 1, pp. 69-81, 2006.

[6] Loper, E.J., and Lynch, D.D., "The HRG: A new low-noise inertial rotation sensor," 16th Joint Services Data Exchange for Inertial Systems, Los Angeles, CA, Nov. 1982.

[7] Lynch, D.D., "Hemispherical Resonator Gyro," in Ragan, R.R. (ed.) "Inertial technology for the future," IEEE Trans. on Aerospace and Electronic Systems, AES-20, 4, pp. 414-444, 1984. 